\title{
Phenotypic Characterization and Antimicrobial Susceptibility of Blood Borne Pathogens in a Tertiary Care Center, Ujjain (M.P), India
}

\author{
Komal Singh, Ramesh Agrawal*, Yogyata Marothi and Harshada Shah \\ Department of Microbiology, R.D. Gardi Medical College, Ujjain, M.P. India \\ *Corresponding author
}

\section{A B S T R A C T}

\section{Keywords \\ BacT/alert, Blood stream infection, Blood samples, Bacterial isolate, Septicaemia, Antimicrobial susceptibility. \\ Article Info \\ Accepted: \\ 25 April 2017 \\ Available Online: \\ 10 May 2017}

Blood stream infections can lead to life threatening sepsis and require immediate antimicrobial treatment. Blood culture is an essential tool for the investigation of clinically suspected sepsis. The present study has been conducted to describe the profile of bacterial isolates from blood cultures and their antibiotic resistance. This is prospective study of 273 blood cultures, collected from clinically suspected cases of bacteraemia studied over a period of five months in a tertiary care hospital in Ujjain, M.P. The isolates were identified by standard biochemical tests and antimicrobial resistance patterns were determined by CLSI guidelines. Blood cultures were positive in 70 (25.6\%) patients by BacT alert system. Gram positive organism accounted for $51.4 \%$ cases; most common being Staphylococcus aureus (47.3\%) followed by Enterococci (4.3\%), Of the Gram negative isolates, Klebsiella spp (14.3\%) was the most common followed by Pseudomonas spp. $(8.6 \%)$. Candida albicans was isolated in $2.8 \%$ cases. Gram positive isolates showed high resistance to penicillin (81.8\%) and least resistance to linezolid (18.2\%). Gram negative isolates were found high resistance to amoxy-clav (90\%) and least resistance to Imipenem (20\%). This study provides information on antibiotic resistance of blood isolates. It may be a useful guide for physicians initiating empiric therapy and will help in formulation of antibiotic therapy strategy.

\section{Introduction}

Blood stream infection (BSI) is the major cause of morbidity and mortality globally. Blood stream infections range from selflimiting infections to life threatening sepsis, which requires rapid and appropriate antimicrobial treatment. Hence periodic monitoring of blood culture isolates and determination of susceptibility to antibiotics are necessary to improve the empirical therapy (Chitralekha et al., 2015). Many bacterial pathogens have developed resistance to most of the antibiotics; it has become a serious health problem with many economic and social inferences all over the worlds (Jo
Ann, 2009). Many bacteria have been reported which cause bacteraemia with variation in distribution from place to place (Gohel et al., 2014). Rapid identification and antimicrobial susceptibility testing of the causative agents of bloodstream infections are the most important tasks of the clinical microbiology laboratory, that provide essential information to clinicians for selecting appropriate antimicrobial therapy for patients with bloodstream infections (Lupetti et al., 2009). Automated blood culture system is non invasive continuous monitoring technology that reduces the time 
needed to detect positive blood cultures as well as decreases the specimen handling (Kim et al., 2010). Published guidelines recommend that the interval between the collection of blood and the entry of the bottles into an automated blood culture system should not be longer than 2 or $4 \mathrm{~h}$; also manufacturer instructions indicate that inoculated vials should be transported to the laboratory as quickly as possible (Clinical Laboratory Standards Institute, 2007; Public Health England, 2014). The changing epidemiology and susceptibility patterns of microorganisms emphasize the necessity of constant surveillance of blood stream infections (Muhammad et al., 2013). The present study was done to analyze various organisms causing bacteremia and their antibiotic resistance pattern. This study wide enable using appropriate antibiotic, may decrease the hospital stay and cost of treatment and reduce Mortality.

The main objectives of this study includes, Isolation and identification of pathogens from blood samples by automated 3D BacT/ALERT blood culture system and their antibiotic resistance patterns in a tertiary care center, Ujjain M.P, India.

\section{Materials and Methods}

This study was carried out at the Department of Microbiology, R.D. Gardi Medical College, Ujjain, Central India. A total of 273 blood samples from clinically suspected cases of septicemia were studied during a 5 months period from Oct 2016 to Feb 2017. Blood samples were collected by using strict aseptic precautions and inoculated immediately into BacT/ALERT FA plus and PF plus aerobic blood culture bottles. After collection these bottles were immediately incubated in BacT/ALERT 3D (bioMerieoux) - a fully automated blood culture system for detection of growth in blood culture. The negative results were followed up to 7days and final report was issued. The BacT/ALERT automatically gives a signal alert. The positive bottles were then subculture on blood, MacConkey and chocolate agar. These plates were incubated aerobically at $37 \mathrm{C}$ and examined after 18-24 hours. Final identification was done by colony characteristics, Gram's staining, motility testing (hanging drop preparation) and routine biochemical test (Catalase, coagulase, indole, methyl red, citrate, urease, Triple sugar iron, PPA, and oxidase testing). Fungal isolate was identified by Gram's staining showing gram positive budding yeast cells and germ tube testing. Antimicrobial susceptibility testing of bacterial isolates was done by the KirbyBauer disc diffusion method using Muller Hinton agar media as per CLSI guidelines.

\section{Results and Discussion}

During the study period, 273 blood cultures were analyzed of which $70(25.6 \%)$ were positive and 203 (74.4\%) were culture negative (Figure 1). Amongst total samples $147(53.8 \%)$ were male and $126(46.2 \%)$ were female patients. The age range varies from 1 month to 87 years. The incidence of blood culture samples was the highest $(26.4 \%)$ in 1 10 years age group followed by 0-1 yrs $(21.3 \%)$. Detail of age groups distribution was given in (Table 1 and Figure 2). Blood samples were collected from different wards and ICUs in the hospital. Maximum number (37.7\%) of blood samples were obtained from pediatrics wards. Details are given in (Table $2)$. Out of 70 blood culture positive isolates, $36(51.4 \%)$ were Gram positive organisms, 32 $(45.7 \%)$ were Gram negatives organisms, while $2(2.8 \%)$ isolates were fungi (Candida spp.). We have also isolate 3 gram positive bacilli (bacillus) and 1 coagulase negative staphylococcus (CoNS) were probably skin contaminant excluded from the positive isolates. Detail description of isolates was 
shown in (Table 3 and Figure 3). Staph aureus $(47.2 \%)$ was the predominant organism in all bacterial isolates followed by Klebsiella spp (14.3\%). Antibiotic resistance pattern of the gram positive organism are shown in (Table 4). Resistance ranges from $18.2 \%$ to $81 \%$. Staph aureus exhibit most resistance to penicillin $(81.8 \%)$ followed by cefoxitin (MRSA) $63.3 \%$ and least resistance to linezolid (18.2\%). Among gram negative organisms Klebsiella was the predominant isolate which exhibit most resistance to amoxclav $(90 \%)$ followed by Amikacin $(80 \%)$ and least resistance to imipenem (20\%). Pseudomonas exhibit most resistance to Cefepime $(66.6 \%)$ followed by Ceftazidime $(50 \%)$ and least resistance to imipenem andAmikacin (16.6\%). Detail description of antibiotic resistance pattern of gram negative organisms is shown in (Table 5).

The findings are matching these respected by sepsis is one of the leading causes of death, and rapid identification of blood stream infection is mandatory to perform adequate antibiotic therapy. In the present study a total of 273 blood culture samples were collected and analyzed, of which $70(25.6 \%)$ were positive by BacT/Alert system. which is quite similar to Sahoo et al., (2016) and Alam et al., (2011) but quite lower Kavitha et al., (2010) and Maimoona et al.,(2014). Majority of the patients $(26.4 \%)$ were in the $1-10$ years age group. Maximum number $(37.7 \%)$ of blood culture came from pediatrics ward. The incidence of Gram-positive organism was $(51.4 \%)$ while $(45.7 \%)$ were Gram-negative organisms which was quite similar to Kalpesh et al., (2014) and China et al., (2013), but in other studies like Maimoona et al., and Ayobola et al., (2011) Gram-negative organisms have taken over Gram-positive organisms in hospital settings. This indicates that infections by Gram-positive organisms constitute a significant threat to bacteremia and septicemia in our hospital setup and the spectrum of organisms is subject to geographical alterations. Among Gram positive organism staphylococcus aureus was the commonest $(47.2 \%)$ isolate followed by Enterococcus $(4.3 \%)$ which was quite similar to study by Kalpesh et al., (2014) and Anbumani et al., (2008).

While Klebsiella spp was the most predominant $(14.3 \%)$ isolate among gram negative organism which was in accordance with Panday et al., (2017).

Table.1 Age and gender wise distribution of blood culture of sepsis patients

\begin{tabular}{|l|l|l|l|}
\hline Age group & Total & Male & Female \\
\hline 1month - 1year & 58 & 28 & 30 \\
\hline$>1-10$ year & 72 & 43 & 29 \\
\hline 11-20 year & 40 & 21 & 19 \\
\hline 21- 30 year & 23 & 14 & 9 \\
\hline 31- 40 year & 18 & 11 & 7 \\
\hline 41-50 year & 25 & 13 & 12 \\
\hline 51- 60 year & 16 & 9 & 7 \\
\hline$>61$ year & 21 & 8 & 13 \\
\hline Total & 273 & 147 & 126 \\
\hline
\end{tabular}


Table.2 Blood samples collected from different wards and ICUs

\begin{tabular}{|c|c|c|}
\hline Word & Number of cases & Percentage (\%) \\
\hline NICU & 10 & 3.7 \\
\hline MICU & 19 & 6.9 \\
\hline PICU & 60 & 21.9 \\
\hline SICU & 12 & 4.4 \\
\hline P/W & 103 & 37.7 \\
\hline MEDICINE & 29 & 10.7 \\
\hline SURGERY & 7 & 2.5 \\
\hline OTHER ICUs & 24 & 8.9 \\
\hline ORTHO & 9 & 3.3 \\
\hline
\end{tabular}

Table.3 Organisms isolated from positive blood samples

\begin{tabular}{|l|c|c|}
\hline Organism & Number of positive cases & Percentage \% \\
\hline Staphylococcus & 33 & 47.2 \\
\hline Enterococcus & 3 & 4.3 \\
\hline Pseudomonas spp. & 6 & 8.5 \\
\hline E. coli & 4 & 5.7 \\
\hline Klebsiella spp. & 10 & 14.3 \\
\hline Citrobacter spp. & 3 & 4.3 \\
\hline Enterobacter & 4 & 5.7 \\
\hline Candida & 2 & 2.8 \\
\hline Non-fermenter GNB & 5 & 7.2 \\
\hline Total & 70 & $100 \%$ \\
\hline
\end{tabular}

Table.4 Antibiotic resistant pattern of gram positive bacteria

\begin{tabular}{|l|c|c|c|c|}
\hline \multirow{2}{*}{ Antibiotic drugs } & \multicolumn{2}{|c|}{ Staph. aureus $(\mathbf{n = 3 3 )}$} & \multicolumn{2}{c|}{ Enterococcus $(\mathbf{n}=3)$} \\
\cline { 2 - 5 } & $\begin{array}{c}\text { No of resistance } \\
\text { isolates }\end{array}$ & $\begin{array}{c}\text { Percentage } \\
(\mathbf{\%})\end{array}$ & $\begin{array}{c}\text { No of resistance } \\
\text { isolates }\end{array}$ & $\begin{array}{c}\text { Percentage } \\
(\%)\end{array}$ \\
\hline Penicillin & 27 & 81.8 & 2 & 66.6 \\
\hline Cefoxitin & 21 & 63.6 & - & - \\
\hline Cotrimoxazole & 17 & 51.5 & - & - \\
\hline Erythromycin & 19 & 57.6 & - & - \\
\hline Clindamycin & 14 & 42.4 & - & - \\
\hline Tetracycline & 7 & 21.2 & - & 0 \\
\hline Vancomycin & 12 & 36.4 & 1 & 63.3 \\
\hline Linezolid & 6 & 18.2 & 0 & 33.3 \\
\hline Ampicillin & - & - & 2 & \\
\hline $\begin{array}{l}\text { High level } \\
\text { Gentamycin }\end{array}$ & - & - & 1 & \\
\hline
\end{tabular}


Table.5 Antibiotic resistant pattern of gram negative bacteria

\begin{tabular}{|l|c|c|c|c|c|c|}
\hline \multirow{2}{*}{ Antibiotic drugs } & \multicolumn{2}{|l|}{ E.coli $(\mathbf{n}=\mathbf{4})$} & \multicolumn{2}{l|}{ Pseudomonas $(\mathbf{n}=6)$} & \multicolumn{2}{l|}{ Klebsiella $(\mathbf{n}=10)$} \\
\cline { 2 - 7 } & Resistance No & $(\boldsymbol{\%})$ & Resistance No & $(\%)$ & Resistance No & $(\%)$ \\
\hline Amikacin & 1 & 25 & 1 & 16.6 & 8 & 80 \\
\hline Aztronem & - & & 2 & 33.3 & - & - \\
\hline Piperacillin & 3 & 75 & 2 & 33.3 & 3 & 30 \\
\hline Cefepime & 3 & 75 & 4 & 66.6 & 7 & 70 \\
\hline Ceftazidime & 2 & 50 & 3 & 50 & 6 & 60 \\
\hline Ciprofloxacin & 3 & 75 & 2 & 33.3 & 4 & 40 \\
\hline Imipenem & 1 & 25 & 1 & 16.6 & 2 & 20 \\
\hline Amoxy-clav & 4 & 100 & - & - & 9 & 90 \\
\hline $\begin{array}{l}\text { Piperacillin } \\
\text { tazobactum }\end{array}$ & 1 & 25 & 2 & 33.3 & 5 & 50 \\
\hline
\end{tabular}

Fig.1 blood culture results $(\mathrm{n}=273)$ by BacT alert system

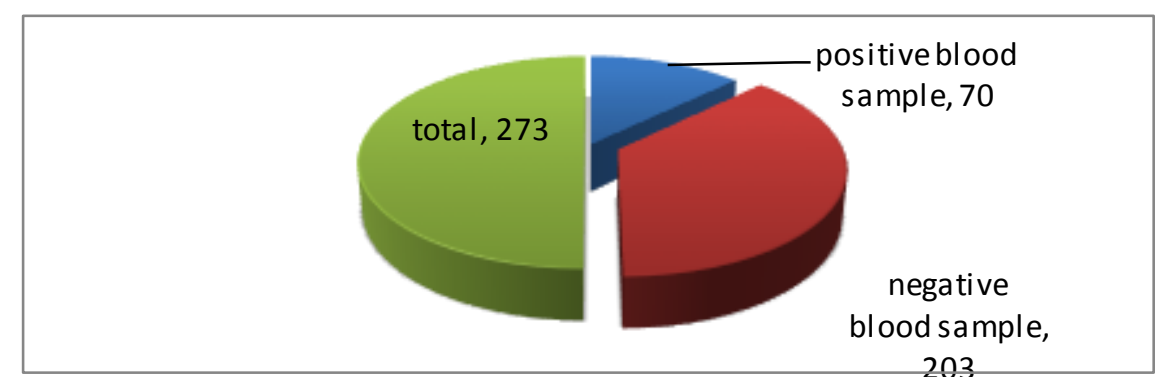

Fig.2 Age and gender wise distribution of blood culture cases

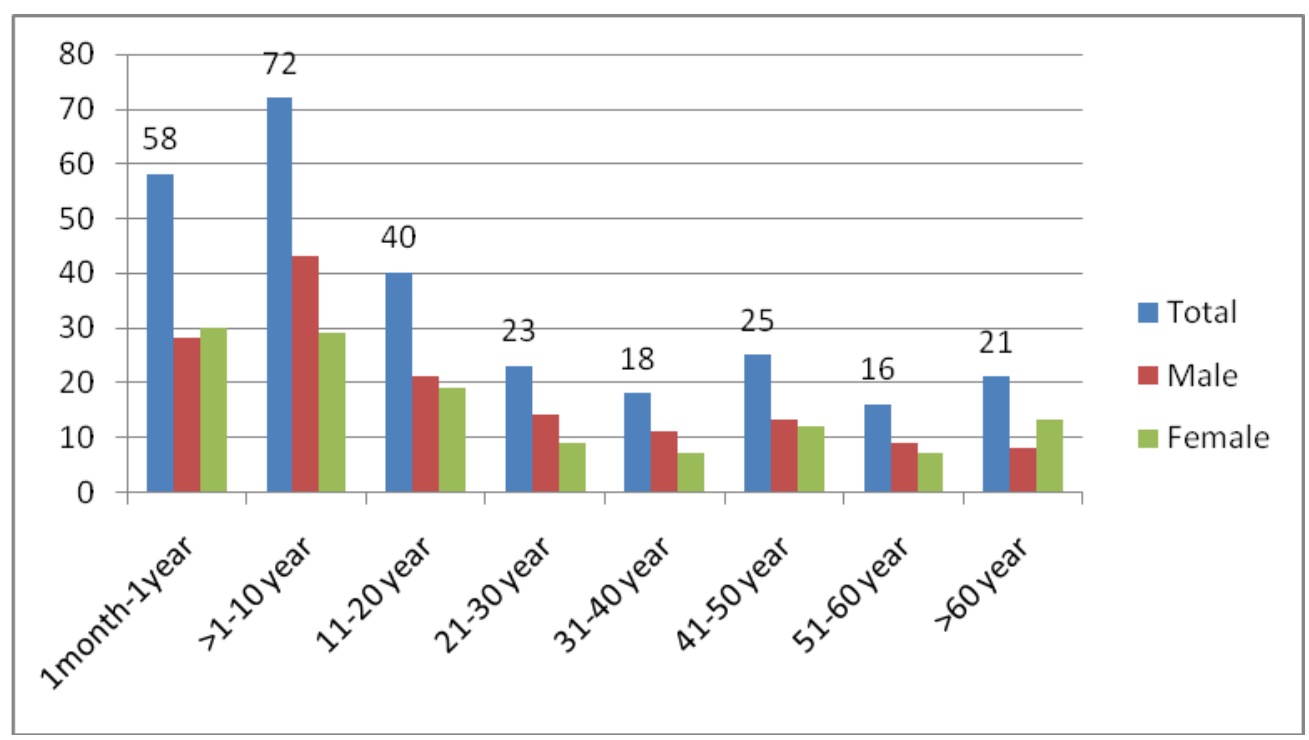


Fig.3 Organisms isolated from automated blood culture

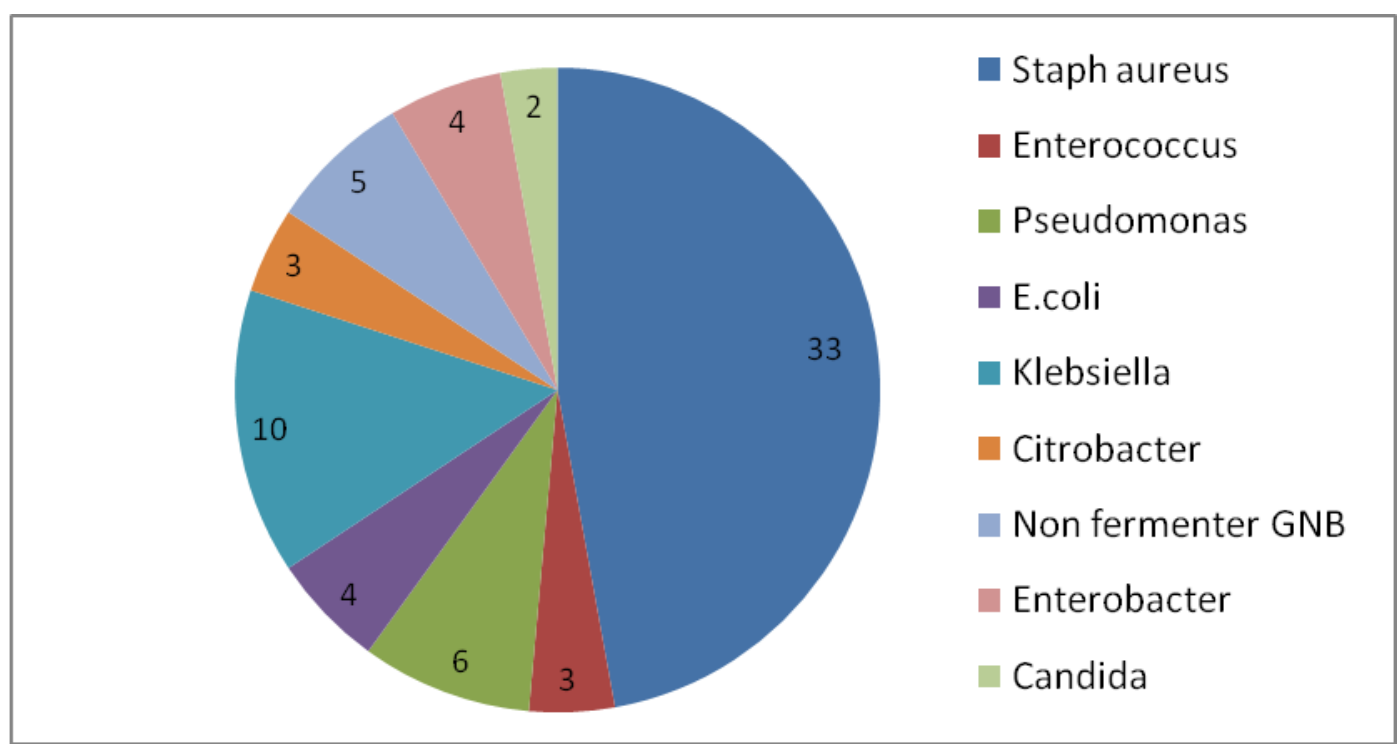

Higher prevalence of antimicrobial resistance was noted in this study, especially in gram negative organism. This might be due to indiscriminate use of antibiotics in hospital. Most of the gram negative organisms were multidrug resistance with a very high resistance to beta-lactam antibiotics and least resistance to Imipenem. The overall resistance of gram positive organism was from 0 to $81.8 \%$, and for gram negative organism from 0 to $100 \%$ these results are in concordance with Araya et al., (2015) which was $0-83 \%$ and $0-100 \%$ for gram positive and negative, respectively. Staphylococcus aureus showed high resistance to cefoxitin (MRSA) which is quite similar to Garg et al., (2007). E. coli and Klebsiella was showed high resistance to amoxy-clav which was accordance to Veena mangunath et al., (2015), and least resistance to imipenem accordance to Aziz japoni et al., (2008).

In the current study fungal septicaemia caused by Candida albicans was observed $2.8 \%$ which was quite similar with the Panday et al., (2017) they have observed 3\% Candida albicans in their study. The rise in antibiotic resistance in blood isolates emphasizes the importance of sound hospital infection control, rational prescribing policies, and the need for awareness to use antimicrobial drugs.

It may be concluded from the study that early diagnosis and appropriate treatment of BSIs should be based on the current knowledge of bacterial profile and antibiotic resistance pattern, which should be provided by microbiology laboratory from time to time. We observed that Staphylococcus aureus and organisms belonging to Enterobacteriaceae family are the leading causes of septicemia. Increasing incidence of drug resistant organisms like MRSA and multidrug resistance gram negative bacilli raises serious concerns and mandates strict antibiotic policy to prevent emergence and spread of antibiotic resistance. We hope that these results could support microbiologists, clinicians and hospital managers in the identification and implementation of strategic targeted actions to coordinate infection control interventions and antimicrobial policies in order to decrease the rate the emergence of resistance and minimize mortality of septicaemic patients. 


\section{References}

Alam, M.S., Pillai, P.K., Kapur, P., Pillai, K.K. 2011. Resistant patterns of bacteria isolated from bloodstream infections at a university hospital in Delhi. J. Pharm. Bioallied Sci., 3: 525-30.

Anbumani, N., et al. 2008. "Distribution and antimicrobial susceptibility of bacteria isolated from blood cultures of hospitalized patients in a tertiary care hospital, Indian J. Practicing Doctor, vol. 5 , no. 2 , pp. $1-7$.

Araya Gebreyesus Wasihun, Letemichael Negash Wlekidan, Senay Aregawi Gebremariam, Tsehaye Asmelash Dejene, Abadi Luel Welderufael, Tadesse Dejenie Haile and Saravanan Muthupandian. 2015. Bacteriological profile and antimicrobial susceptibility patterns of blood culture isolates among febrile patients in Mekelle Hospital, Northern Ethiopia, Springerplus, 4: 314. Published online 2015 Jul 3. doi: 10.1186/s40064-015-1056-x

Ayobola, E.D., et al. 2011. "Study of prevalence and antimicrobial susceptibility of blood culture bacterial isolates," Malaysian J. Microbiol., vol. 7, no.2, pp.78-82.

Aziz Japoni, Shohreh Farshad, Abdolvahab Alborzi, Mehdi Kalani, Nouradin Rafaatpour, Barat Oboodi and Bahman Pourabbas, 2008. Epidemiology and Antibacterial Susceptibility Patterns of Bloodstream Infections, 2001-2004: An Experience with BACTEC 9240 in Southern Iran. Pakistan J. Biol. Sci., 11: $422-427$.

China, D., and V. Gupta. 2013. "Bacteriological profile and antimicrobial susceptibility pattern of blood isolates from a tertiary care hospital in North India," Int. $J$.
Pharmaceutical Res. Biosci., vol. 2, no. 2, pp. 24-35.

Chitralekha Saikumar, Bindu, Kiran Madhusudhan, Praveena, R., Illamani, V. 2015. Aerobic Microbial Profile and Antibiotic Susceptibility of Blood Isolates in a Tertiary Care Center, Int. $J$. Pharm. Sci. Rev. Res., 34(2), Article No. 23, Pages: 135-137.

Clinical Laboratory Standards Institute. 2007. Principles and procedures for blood cultures; approved guideline CLSI document M47-A. CLSI, Wayne, PA.

Debananda Sahoo, Lalatendu Mohanty, S.S. Panda, S.N., Mishra. 2016. Bacteriological Analysis of Blood Culture Isolates in Patients with Sepsis in A Tertiary Care Hospital of Eastern India, IJCMR, Volume 3 | Issue 12 | December 2016 | ICV (2015): 77.83 | ISSN (Online): 2393-915X; (Print): 2454-7379.

Garg, A., et al. 2007. "Bacteriological profile and antimicrobial resistance of blood culture isolates from a university hospital," J. Indian Academy of Clin. Med., vol. 8, no. 2, pp. 139-143.

Gohel, K., Jojera, A., Soni, S., Gang, S., Sabnis, R., Desai, M. 2014. Bacteriological profile and drug resistance patterns of blood culture isolates in a tertiary care nephrourology teaching institute. Biomed. Res. Int., 153747. doi: 10.1155/2014/153747

Jo Ann, D. 2009. Antibiotic resistance: the ongoing challenge for effective drug therapy. JAAPA, 22(3): 18-22A.

Kalpesh Gohel, et al. 2014. Bacteriological Profile and Drug Resistance Patterns of Blood Culture Isolates in a Tertiary Care Nephrourology Teaching Institute, Hindawi Publishing Corporation BioMed Res. Int., Article ID 153747, 5 pages http://dx.doi.org/10.1155/2014/153747. 
Kavitha, P., et al. 2010. "Bacteriological profile and antibiogram of blood culture isolates in a pediatric care unit," $J$. Laboratory Physicians, vol. 2, pp. 8588.

Kim, K.E. and J.Y. Han, 2010. Evaluation of the clinical performance of an automated pro calcitonin assay for the quantitative detection of bloodstream infection. Korean. J. La. Med., 30: 153159. DOI: $10.3343 / \mathrm{kjlm} .2010 .30 .2 .153$

Lupetti, et al. 2009. Rapid identification and antimicrobial susceptibility testing of Gram-positive cocci in blood cultures by direct inoculation into the $\mathrm{BD}$ Phoenix system, Clin. Microbiol. Infect., Volume 16, Issue 7.

Maimoona Mustafa and Syed Laeeq Ahmed. 2014. Bacteriological profile and antibiotics susceptibility pattern in neonatal septicemia in view of emerging drug resistance, $J$. Med. Allied Sci., 4(1): 02-08 www. jmas. in Print ISSN: 22311696 Online ISSN: 2231170X.

Manjusha Pandey, Devendra Niranjan and R.C., Pande. 2017. Bacteriological Profile and Antimicrobial Resistance of Blood Culture Isolates from a 350 bedded Hospital Lucknow, India. Int. J. Curr. Microbiol. App. Sci., 6(1): 184193. doi: http://dx.doi.org/10.20546/ijcmas.2017. 601.023

Mathew, A., et al. 2006. "Performance standards for antimicrobial susceptibility testing," Clinical and Laboratory Standards Institute, vol. 26, supplement 16.

Muhammad Fayez, Irfan Ali Mirza, Aamer Ikram, Aamir Hussain, Tahir Ghafoor and Umer Shujat. 2013. Pathogens causing blood stream infections in immunocompromised patients, $J$. College of Physicians and Surgeons Pakistan, 13, Vol. 23 (12): 848-851

Public Health England. 2014. UK standards for microbiology investigations. Investigation of blood cultures (for organisms other than Mycobacterium Species). Bacteriol., 8: 1-51

Veena Manjunath, Chillargi, C., Prathiba, S., Amarnath, S.K., Hegde, S.M. 2015. Microbial and antimicrobial resistance profile of bloodstream infections: A hospital-based study, Medica Innovatica, Volume 4- Issue 2.

\section{How to cite this article:}

Komal Singh, Ramesh Agrawal, Yogyata Marothi, Harshada Shah. 2017. Phenotypic Characterization and Antimicrobial Susceptibility of Blood Borne Pathogens in a Tertiary Care Center, Ujjain (M.P). Int.J.Curr.Microbiol.App.Sci. 6(5): 2271-2278. doi: https://doi.org/10.20546/ijcmas.2017.605.253 